\title{
The combination of blueberry juice and probiotics reduces apoptosis of alcoholic fatty liver of mice by affecting SIRTI pathway
}

This article was published in the following Dove Press journal:

Drug Design, Development and Therapy

12 May 2016

Number of times this article has been viewed

\author{
Juanjuan Zhu' ${ }^{1,2, *}$ \\ Tingting Ren ${ }^{3, *}$ \\ Mingyu Zhou ${ }^{2}$ \\ Mingliang Cheng ${ }^{2}$
}

'First Hospital Affiliated to Suzhou University, Suzhou, ${ }^{2}$ Department of Infectious Diseases, ${ }^{3}$ Biochemistry Department, Affiliated Hospital of Guiyang Medical College, Guiyang,

People's Republic of China

*These authors contributed equally to this work

\footnotetext{
Correspondence: Mingliang Cheng Department of Infectious Diseases, Affiliated Hospital of Guiyang Medical College, 28 Guiyi Street, Guiyang 550004, Guizhou Province, People's Republic of China

Tel +868515657878

Fax +86 85I 5657879

Email minglianggy@I63.com
}

Purpose: To explore the effects of the combination of blueberry juice and probiotics on the apoptosis of alcoholic fatty liver disease (AFLD).

Methods: Healthy C57BL/6J mice were used in the control group (CG). AFLD mice models were established with Lieber-DeCarli ethanol diet and evenly assigned to six groups with different treatments: MG (model), SI (SIRT1 [sirtuin type 1] small interfering RNA [siRNA]), BJ (blueberry juice), BJSI (blueberry juice and SIRT1 siRNA), BJP (blueberry juice and probiotics), and BJPSI (blueberry juice, probiotics, and SIRT1 siRNA). Hepatic tissue was observed using hematoxylin and eosin (HE) and Oil Red O (ORO) staining. Biochemical indexes of the blood serum were analyzed. The levels of SIRT1, caspase-3, forkhead box protein O1 (FOXO1), FasL (tumor necrosis factor ligand superfamily member 6), BAX, and $\mathrm{Bcl}-2$ were measured by reverse transcription-polymerase chain reaction and Western blotting.

Results: HE and ORO staining showed that the hepatocytes were heavily destroyed with large lipid droplets in MG and SI groups, while the severity was reduced in the CG, BJ, and BJP groups $(P<0.05)$. The levels of superoxide dismutase (SOD), reduced glutathione (GSH), and high-density lipoprotein-cholesterol (HDL-C) were increased in BJ and BJP groups when compared with the model group $(P<0.05)$. In contrast, the levels of aspartate aminotransferase (AST) and alanine aminotransferase (ALT), total triglycerides (TGs), total cholesterol, low-density lipoprotein-cholesterol (LDL-C), and malondialdehyde (MDA) were lower in BJ and BJP groups than in the model group $(P<0.05)$. The level of SIRT1 was increased, while the levels of FOXO1, phosphorylated FOXO1, acetylated FOXO1, FasL, caspase-3, BAX, and Bcl-2 were decreased in CG, BJ, and BJP groups $(P<0.05)$. Meanwhile, SIRT1 silence resulted in increase of the levels of FOXO1, phosphorylated FOXO1, acetylated FOXO1, FasL, caspase-3, BAX, and Bcl-2.

Conclusion: The combination of blueberry juice and probiotics reduces apoptosis in AFLD by suppressing FOXO1, phosphorylated FOXO1, acetylated FOXO1, FasL, caspase-3, BAX, and $\mathrm{Bcl}-2$ via the upregulation of SIRT1.

Keywords: alcoholic fatty liver, blueberry, caspase-3, forkhead box protein O1, probiotics, sirtuin type 1, tumor necrosis factor ligand superfamily member 6

\section{Introduction}

Alcoholic fatty liver disease (AFLD) is a very common disorder because long-term consumption of alcohol in excessive amounts induces clinical manifestations of liver injury. Hepatitis, fibrosis, cirrhosis, and liver cancer develop if the progression of AFLD is not well controlled. ${ }^{1-3}$ The onset of AFLD is increasing in the world. ${ }^{4}$ Treatment with drugs is one of the main methods of controlling AFLD. The drugs commonly used for AFLD therapy are corticosteroids, ${ }^{5}$ tumor necrosis factor- $\alpha$, and so on. ${ }^{6}$ However, all these drugs have serious side effects and cannot be tolerated by all people. ${ }^{7}$ Therefore, 
it is necessary to explore nonpharmaceutical interventions and natural food components for use in AFLD therapy.

The pathogenesis of AFLD is closely related with the oxidative stress caused by alcohol metabolism, which results in the apoptosis of liver cells. ${ }^{8}$ Under normal circumstances, it is critical to maintain the equilibrium between proliferation and apoptosis of the liver cell. Otherwise, an increase in apoptotic cells will cause liver inflammation and necrosis, resulting in alcoholic hepatitis. ${ }^{9}$ Many studies ${ }^{10-12}$ show that the inhibition of hepatic apoptosis can reduce liver injury. Antioxidants can ameliorate ethanol-induced apoptosis in liver cells. ${ }^{13}$

Blueberries constitute a widely distributed species in North America, Europe, Asia, and Africa, and they have the highest antioxidant capacity. ${ }^{14}$ Ultra-performance liquid chromatography-electrospray ionization quadrupole timeof-flight/mass spectrometry analysis shows that blueberries are rich in quinic acid $(203-3,614 \mu \mathrm{g} / \mathrm{mL})$, chlorogenic acid (20.0-346.8 $\mu \mathrm{g} / \mathrm{mL})$, and rutin $(0.00-26.88 \mu \mathrm{g} / \mathrm{mL})$, and the contents varied depending on the genotypes. ${ }^{15}$ Quinic acid shows high antioxidant activities by inducing the production of the antioxidants tryptophan ${ }^{16}$ and nicotinamide ${ }^{17}$ in the gastrointestinal tract and by improving DNA repair. On the other hand, quinic acid can increase the levels of tryptophan and nicotinamide by affecting the microflora in the gastrointestinal tract, as well as providing the essential metabolic ingredients for people's health. ${ }^{18}$ Previous work has shown that chlorogenic acid treatment ameliorates the intestinal mitochondrial injury induced by $\mathrm{H}_{2} \mathrm{O}_{2}$; meanwhile, the levels of reactive oxygen species, 8-hydroxy-2-deoxyguanosine, and cytochrome $\mathrm{c}$ were reduced. ${ }^{19}$ Rutin has been approved to have antioxidant and antibacterial activities. ${ }^{20}$ Moreover, probiotics can reduce liver damage and inhibit hepatic apoptosis. ${ }^{21}$ Considering the importance of gut microbiota in the development of AFLD, the function of probiotics in AFLD therapy has been recognized. Probiotic intervention inhibits the progression of AFLD in animal models and patients. $^{22}$ Thus, blueberry juice and probiotics may have some complementary functions in the treatment of AFLD.

However, the synergistic effects of a combination of blueberry juice and probiotics on AFLD remain unclear. Sirtuin type 1 (SIRT1), an NAD-dependent deacetylase, plays an important role in preventing metabolic diseases. ${ }^{23,24}$ SIRT1 deletion in mice increases hepatic steatosis and inflammation. ${ }^{25,26}$ Serum SIRT1 has been reported to be associated with fatty liver infiltration. ${ }^{27}$ Many studies have shown that SIRT1 affects energy metabolism, ${ }^{28,29}$ oxidative injury, ${ }^{30}$ and apoptosis. ${ }^{31}$ SIRT1 has been reported to affect the promoter of p66shc. ${ }^{32}$ Salvianolic acid A can protect rats against apoptosis in liver injury by affecting the SIRT1/p66shc pathway. ${ }^{33}$
All these results suggest that SIRT1 is an important regulator in liver injury. Therefore, to understand the molecular mechanism of the effects of blueberry and probiotics on AFLD, an AFLD model was established successfully in this study, and the inhibitory mechanisms were elucidated with SIRT1 small interfering RNA (siRNA) lentivirus. SIRT1, ${ }^{34,35}$ an apoptosis inhibitor, reduces the levels of the apoptosis factors caspase- $3,{ }^{36}$ $\mathrm{BAX},{ }^{37} \mathrm{Bcl}-2,{ }^{38}$ forkhead box protein $\mathrm{O} 1$ (FOXO1), ${ }^{39}$ and tumor necrosis factor ligand superfamily member 6 (FasL). ${ }^{40}$ On the other hand, FOXO1 is a phosphorylated protein, and the phosphorylation of FOXO1 by Akt leads to nuclear export and inhibition of the transcription activity. ${ }^{41}$ Additionally, SIRT1 can inhibit FOXO1 by deacetylation. ${ }^{42}$ Therefore, the level of phosphorylated FOXO1 and the acetylation status of FOXO1 were determined.

\section{Materials and methods \\ Animals}

All the protocols in this study were approved by the animal care and ethical committee of Suzhou University. A total of 56 female C57BL/6J mice, 6-8 weeks of age and with body weight $25 \pm 5 \mathrm{~g}$, were purchased from the Experimental Animal Center, Third Military Medical University (Animal license, SYXK Qian 2012-0001; ethics license: 1403070). All animal-handling procedures were performed according to the Guide for the Care and Use of Laboratory Animals of the National Institutes of Health and followed the guidelines of the Animal Welfare Act. All animals were housed in a 12 hour light/dark cycle and had constant access to food and water.

\section{Materials}

Blueberry juice (Vaccinium spp.) was purchased from Majiang Blueberry Plant (Guiyang, People's Republic of China) and preserved at $-20^{\circ} \mathrm{C}$ until use. The ingredients of blueberry juice, including anthocyanins, trace elements, and vitamins, were determined according to a previous report. ${ }^{43}$ The mixed probiotics containing Bifidobacterium, Lactobacillus bulgaricus, and Streptococcus thermophilus were purchased from Inner Mongolia Double Odd Pharmaceutical Co (OTCS19980004; Inner Mongolia, People's Republic of China). ${ }^{44}$

RNA extraction and purification kit was purchased from Biomiga Inc (R6311; Biomiga, CA, USA), SYBR Premix Ex Taq II (Tli RNaseH Plus) from Clontech (Mountain View, CA, USA), and PrimeScript ${ }^{\mathrm{TM}}$ RT Reagent Kit with gDNA Eraser (Perfect Real Time) from Takara Bio Inc (RR047A; Dalian, People's Republic of China). SIRT1, caspase-3, FOXO1, and FasL primers were synthesized by Takara Bio Inc. SIRT1, caspase-3, FOXO1, FasL, and BAX rabbit 
anti-mouse antibodies (ab39670, ab2302, ab179450, ab68338, and ab10813) were purchased from Abcam (Cambridge, MA, USA). Anti-phosphorylated-FOXO1 (Ser256) Antibody (catalog no 9461) and Bcl-2 antibody (catalog no D17C4) were purchased from CST (Shanghai) Biological Reagents (Shanghai, People's Republic of China). Anti-acetylated FOXO1 (Ac-FoxO1) antibody (catalog no sc-49437) was purchased from Santa Cruz Biotechnology (Shanghai) Co Ltd (Shanghai, People's Republic of China). Goat anti-rabbit secondary antibody was from Shanghai Genetic Engineering Company (GK500710; Shanghai, People's Republic of China). Glyceraldehyde-3-phosphate dehydrogenase (GAPDH) rabbit anti-mouse antibody was procured from Wuhan Boster Biotechnology Co (21260-1-ap; Wuhan, People's Republic of China). Bicinchoninic acid protein quantification kit was purchased from Biomiga Inc (PW0104). Electrochemiluminescence (ECL) kit was from Tupper Company (WBKLS0100, Farmersville, MA, USA). Mouse Sirt-1, caspase-3, FOXO1, and FasL enzyme-linked immunosorbent assay kits were purchased from Shanghai Comissariado (Shanghai, People's Republic of China).

\section{The establishment of AFLD mouse model}

Eight C57/6J mice were fed only Lieber-DeCarli '82 diet (F1259SP) as a control group. Forty C57/6J mice were fed Lieber-DeCarli diet (F1258SP) along with alcohol for 10 days. Lieber-DeCarli ' 82 and Lieber-DeCarli diets were purchased from Bio-Serve (Flemington, NJ, USA).

\section{SIRTI siRNA}

SIRT1 siRNA sequence(GCAGGTTGCAGGAATCCAAAG) was synthesized and linked with lentivirus vector using a carrier LV3 (H1/GFP and Puro) from Shanghai GenePharma Technology Co Ltd (Shanghai, People's Republic of China). The sequence was linked to the pshRNA-H1-Luc lentivector to construct pshRNA-H1-Luc-SIRT1-siRNA. The constructed vector was cotransfected into $293 \mathrm{~T}$ cells by Lipofectamine ${ }^{\text {TM }} 2000$ (Thermo Fisher Scientific, Waltham, MA, USA) and pPACK Packaging Plasmid Mix (System Biosciences, Shanghai, People's Republic of China). After 48-hour culture, viral supernatant was collected and the titer was calculated with diluted lentivirus. Twenty-four AFLD mice were intra-articularly injected with $100 \mu \mathrm{L}$ supernatants containing the lentivirus-carrying SIRT1 siRNA $\left(1 \times 10^{8}\right.$ virus titer per injection, two times/day) for 10 days.

\section{Animal groups}

All mouse models with AFLD were evenly and randomly assigned to six groups (Figure 1) as follows: model group (MG);
1) blueberry juice group $(\mathrm{BJ})$, in which the mice were fed blueberry juice $(1.5 \mathrm{~mL} / 100 \mathrm{~g})$ for 10 days; ${ }^{45}$ 2) blueberry juice and probiotic bacteria group (BJP), in which the mice were fed blueberry juice and probiotics (blueberry juice: $1.5 \mathrm{~mL} / 100 \mathrm{~g}$; $20 \mathrm{~mL} / 100 \mathrm{~g}$ probiotics were used.) for 10 days; 3) SIRT1 siRNA group (SI), in which the SIRT1 gene was silenced; 4) blueberry juice and SIRT1 siRNA group (BJSI), in which the SIRT1 gene was silenced in the mice and the mice were fed blueberry juice (1.5 mL/100 g) for 10 days; and 5) blueberry juice, probiotics, and SIRT1 siRNA group (BJPSI), in which the SIRT1 gene was silenced in the mice and the mice were fed blueberry juice and probiotics (blueberry juice, $1.5 \mathrm{~mL} / 100 \mathrm{~g}$; the concentrations of probiotics were $250 \mathrm{mg} / 100 \mathrm{~mL}$ and $20 \mathrm{~mL} / 100 \mathrm{~g}$ probiotics were used) for 10 days. Meanwhile, 8 healthy mice were used as a control group (CG).

\section{Histological analysis}

All mice were anesthetized and sacrificed. Blood was taken and centrifuged at $5,000 \times g$ for 10 minutes, and serum was separated and stored at $-80^{\circ} \mathrm{C}$. The liver tissues were taken and fixed in 10\% neutral formalin, cut into paraffin-embedded sections, and stained with hematoxylin and eosin (HE) and Oil Red O. Each slice was randomly selected in five microscopic fields ( $\times 400)$. One hundred cells were counted under Olympus BX41 image system (OLYMPUS Company, Tokyo, Japan).

\section{Flow cytometry analysis}

Flow cytometry analysis was performed to measure the apoptotic status of liver cells. First, the liver cells were separated based on the method introduced in a report. ${ }^{46}$ Briefly, $100 \mu \mathrm{L}$ cells at $5 \times 10^{5} / \mathrm{mL}$ were transferred into $5 \mathrm{~mL}$ flow tubes. Annexin V/fluorescein isothiocyanate (FITC) $(5 \mu \mathrm{L})$ was added, and apoptotic rates were detected based on the fluorescence of Annexin V/FITC with a flow cytometer (Coulter Epics XL; Beckman Coulter, Fullerton, CA, USA).

\section{Biochemical analysis}

For biochemical analysis, serum samples were used. Oxidative stress is associated with the development of AFLD. Superoxide dismutase (SOD), ${ }^{47}$ aspartate aminotransaminase (AST), ${ }^{48}$ alanine aminotransferase (ALT) ${ }^{48}$ reduced glutathione (GSH), ${ }^{49}$ and malondialdehyde (MDA) all have antioxidant activities. ${ }^{50}$ The levels of AST and ALT were determined by a Biochemical Analyzer (Siemens Advia 1650; Siemens, Bensheim, Germany). The levels of SOD and GSH were measured according to previous reports. ${ }^{51,52}$ The level of MDA was measured with an MDA kit (Nanjing Jiancheng Bioengineering Institute, Nanjing, People's Republic of China). 


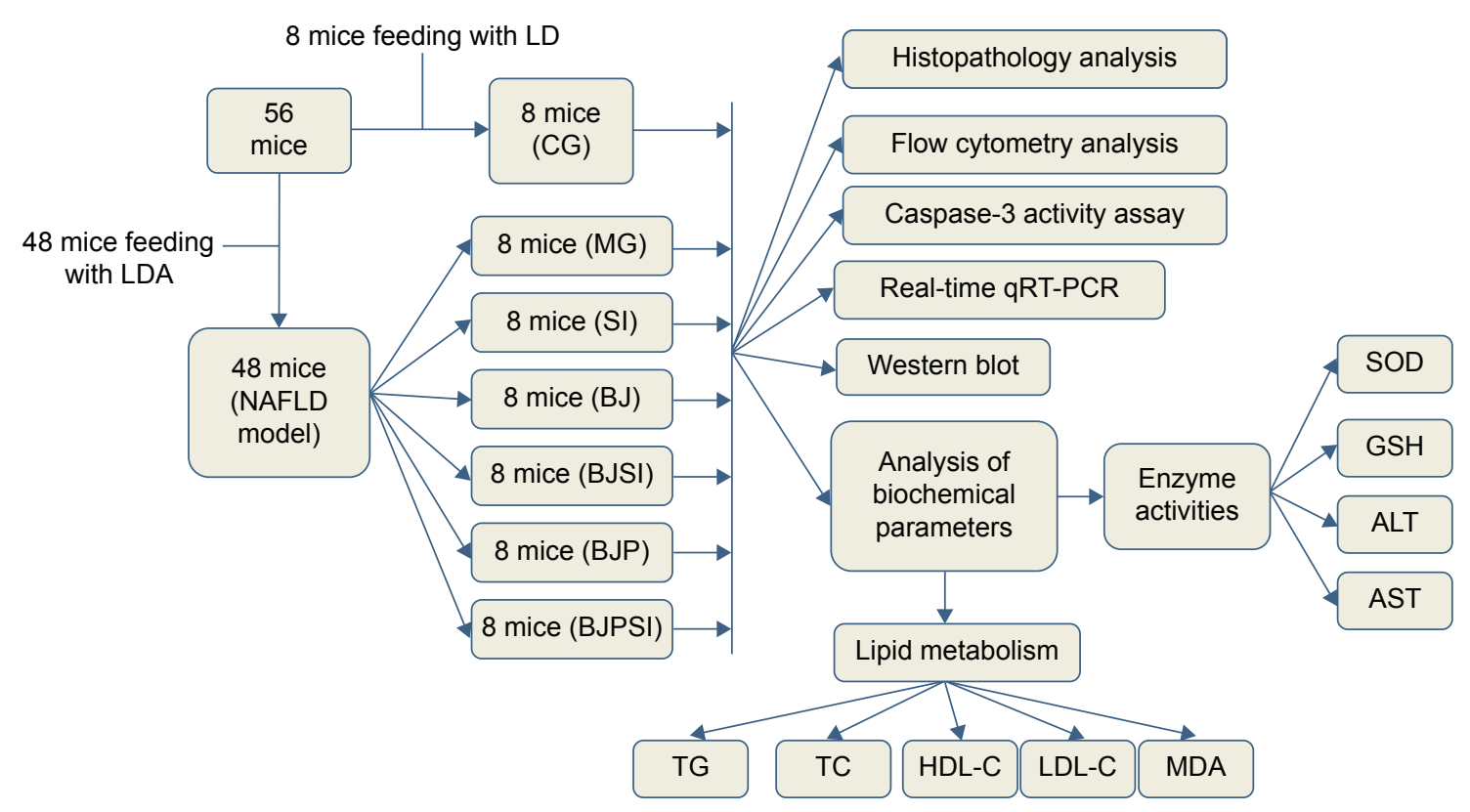

Figure I The flow chart of the study.

Notes: The AFLD model was established using LDA. CG is the control group, in which the mice were fed with Lieber-DeCarli (LD) diet; MG is the model group, in which the mice were fed with LD diet and alcohol; SI is a model group, in which SIRTI gene was silenced in the model mice; $\mathrm{B}$ ) is a model group, in which the model mice were fed with blueberry juice; BJSI is a model group, in which SIRTI gene was silenced in the model mice and the mice were fed with blueberry juice. BJP is a model group, in which the model mice were fed with blueberry juice and probiotics; BJPSI is a model group, in which SIRTI gene was silenced in model mice and the mice were fed with blueberry juice and probiotics.

Abbreviations: SOD, superoxide dismutase; GSH, reduced glutathione; AST, aspartate aminotransferase; ALT, alanine aminotransferase; TG, total triglyceride; TC, total cholesterol; HDL-C, high-density lipoprotein-cholesterol; LDL-C, low-density lipoprotein-cholesterol; MDA, malondialdehyde; LDA, Lieber-DeCarli diet and alcohol; MG, model group; SI, SIRTI siRNA group; SIRTI, sirtuin type I; BJ, blueberry juice group; BJSI, blueberry juice and SIRTI siRNA group; BJP, blueberry juice and probiotics group; BJPSI, blueberry juice, probiotics, and SIRTI siRNA group; qRT-PCR, quantitative reverse transcription-polymerase chain reaction; NAFLD, nonalcoholic fatty liver disease.

AFLD is generally characterized by accumulation of lipids. A standard lipid panel includes total triglycerides (TGs), total cholesterol (TC), high-density lipoprotein-cholesterol (HDL-C), and low-density lipoprotein-cholesterol (LDL-C). ${ }^{53,54}$ The levels of TGs, TC, HDL-C, and LDL-C were measured using a biochemical analyzer (7020; Hitachi High-Technologies, Shanghai, People's Republic of China).

\section{Quantitative reverse transcription- polymerase chain reaction analysis}

Total RNA was isolated, and its concentration was measured on Nanodrop 2000c (Thermo Fisher Scientific). Complementary DNA was synthesized according to the instructions of reverse transcription-polymerase chain reaction (RT-PCR) kits. Quantitative RT-PCR (qRT-PCR) was used to detect the mRNA levels of SIRT1, caspase-3, FOXO1, FasL, BAX, and $B c l-2$ genes using the primers listed in Table 1. GAPDH was used as an internal control to standardize the copy number $(\mathrm{Ct}$ value) of each sample. qRT-PCR was performed on a DA7600 real-time fluorescence detection system (Zhongshan Da An Gene Co, Ltd, Guangzhou, People's Republic of China). The mean $\mathrm{Ct}$ values represent the mRNA levels of these genes.

\section{Western blot analysis}

Western blotting was used to detect the protein levels. All proteins were extracted and the concentrations were measured. An aliquot containing $40 \mu \mathrm{g}$ proteins was taken from each sample, separated by $10 \%$ sodium dodecyl sulfate polyacrylamide gel electrophoresis (SDS-PAGE), and then transferred to a membrane, which was blocked with $5 \%$ nonfat milk. The membrane was incubated with the primary antibody at $4{ }^{\circ} \mathrm{C}$ overnight. Secondary antibody was added and incubated at room temperature for 1 hour. Brown bands were developed after 1-hour ECL exposure, scanned by Gel Doc EQ condensate gel imager, and analyzed by the Quantity software. The expression levels were shown as gray values based on the relative ratio to GAPDH. The results were recorded using a GelDocXR gel imaging system (BIO-RAD Company, Hercules, CA, USA).

\section{Caspase- 3 activity assay}

Liver tissues were lysed using the lysis buffer in the caspase- 3 activity assay kit by incubating for 15 minutes on ice (catalog no C1115; Beyotime Institute of Biotechnology, Beijing, People's Republic of China). Cell lysates were centrifuged at $20,000 \times g$ for 10 minutes at $4^{\circ} \mathrm{C}$, and 
Table I The primers used in qRT-PCR

\begin{tabular}{|c|c|c|c|}
\hline Gene & Primer sequence & Annealing temperature $\left({ }^{\circ} \mathrm{C}\right)$ & Fragment size (bp) \\
\hline \multirow[t]{2}{*}{ GAPDH } & F:5'-GTTGTCTCCTGCGACTTCA3' & 60 & 293 \\
\hline & R:5'-GCCCCTCCTGTTATTATGG3' & & \\
\hline \multirow[t]{2}{*}{ SIRTI } & F:5'-GGAACCTTTGCCTCATCTACAT3' & 60 & 164 \\
\hline & R:5'-ATTCCTTTTGTGGGCGTGG3' & & \\
\hline \multirow[t]{2}{*}{ Caspase-3 } & F:5'-TATCCTGAAATGGGCATATG3' & 60 & 140 \\
\hline & R:5'-TTCCTGACTTGGTATTTCAG3' & & \\
\hline \multirow[t]{2}{*}{ FOXOI } & F:5'-AGTGGATGGTGAAGAGCGT3' & 60 & 324 \\
\hline & R:5'-GCCACTTAGAAAACTGAGACC $3^{\prime}$ & & \\
\hline \multirow[t]{2}{*}{ FasL } & F:5'-GGTGCTTGCTGGCTCACAGT3' & 60 & 88 \\
\hline & R:5'-TTCACGAACCCGCCTCCTC3' & & \\
\hline \multirow[t]{2}{*}{$B A X$} & F:5'-CATCGAGGTGGAGAGCGACG3' & 60 & 140 \\
\hline & R:5'-GTGATGGGACTGAGTCCCGC3' & & \\
\hline \multirow[t]{2}{*}{$\mathrm{BCl}-2$} & F:5'-AGAAGAAGGGAGAATCACAG3' & 60 & 160 \\
\hline & R:5'-CGGCATGATCTTCTGTCAAG3' & & \\
\hline
\end{tabular}

Abbreviations: F, forward; qRT-PCR, quantitative reverse transcription-polymerase chain reaction; R, reverse.

the supernatants were collected. The activity of caspase-3 was measured according to the manufacturer's instructions on the kit. Acetyl-Asp-Glu-Val-Asp-p-nitroaniline (Ac-DEVD-pNA) is cleaved and $p$-nitroaniline (pNA) is released as the chromophore. The absorbance values at $405 \mathrm{~nm}$ stand for the activity of caspase-3. One unit of caspase- 3 is defined as the amount of enzyme that cleaves $1 \mathrm{nmol} \mathrm{pNA} / \mathrm{h}$ at $37^{\circ} \mathrm{C}$.

\section{Statistical analysis}

All the data are presented as mean \pm standard deviation (SD). All the groups were compared using analysis of variance and $t$-test. SPSS 17.0 (SPSS Inc, Chicago, IL, USA) statistical software was used, and $P<0.05$ was considered statistical difference. $P<0.01$ was a statistically significant difference.

\section{Results}

\section{Blueberry juice and probiotics ameliorate AFLD}

A mouse model with AFLD was established, and the severity of AFLD was analyzed by staining with HE and Oil Red O (Figure 2). In healthy animals, liver cells radially encircle a tubular section in the center, and all lipid droplets are of small size. In the AFLD model group, the lobular structure was destroyed and widely distributed, with more lipid droplets when compared with controls. In the SI group, the lobular structure was heavily destroyed and filled with many large lipid droplets when compared with the liver tissues from the model group. The liver injury suggested that an AFLD mouse model was created. The severity of AFLD was reduced, and lipid droplets were decreased in the BJ and BJP groups when compared with the same in MG, SI, BJSI, and BJPSI groups (Figure 2). In the SI group, the structure of the hepatic cord was heavily destroyed and many large lipid droplets were produced when the SIRT1 gene was blocked, suggesting that SIRT1 plays an important role in maintaining the normal structure of liver cells. In the BJ group, the hepatic cords were arranged around a central vein clearly. A fewer number of small-sized lipid droplets were produced. In the BJSI group, the hepatic structure of cords was heavily destroyed and more lipid droplets were produced when SIRT1 was blocked. In the BJP group, the hepatic cords were radially arranged around a central vein and there were fewer lipid droplets. In the BJPSI group, the hepatic structure was destroyed and the lipid droplets were of small size. These findings suggested that the combination of blueberry juice and probiotics attenuates AFLD better than the group fed only blueberry juice.

\section{Liver apoptosis}

Annexin/FITC analysis indicated that the apoptotic rate was the highest in the SI group and the rate was reduced in the BJSI and BJPSI groups when blueberry juice and/or probiotics were used (Figure 3) $(P<0.05)$. In comparison, the apoptotic rate was higher in the MG group than in the $\mathrm{BJ}$ and BJP groups. The rate was reduced in the BJ and BJP groups when blueberry juice and/or probiotics were used (Figure 3) $(P<0.05)$. These results suggested that blueberry juice and/or probiotics consumption reduces the apoptotic rate of liver tissues. 

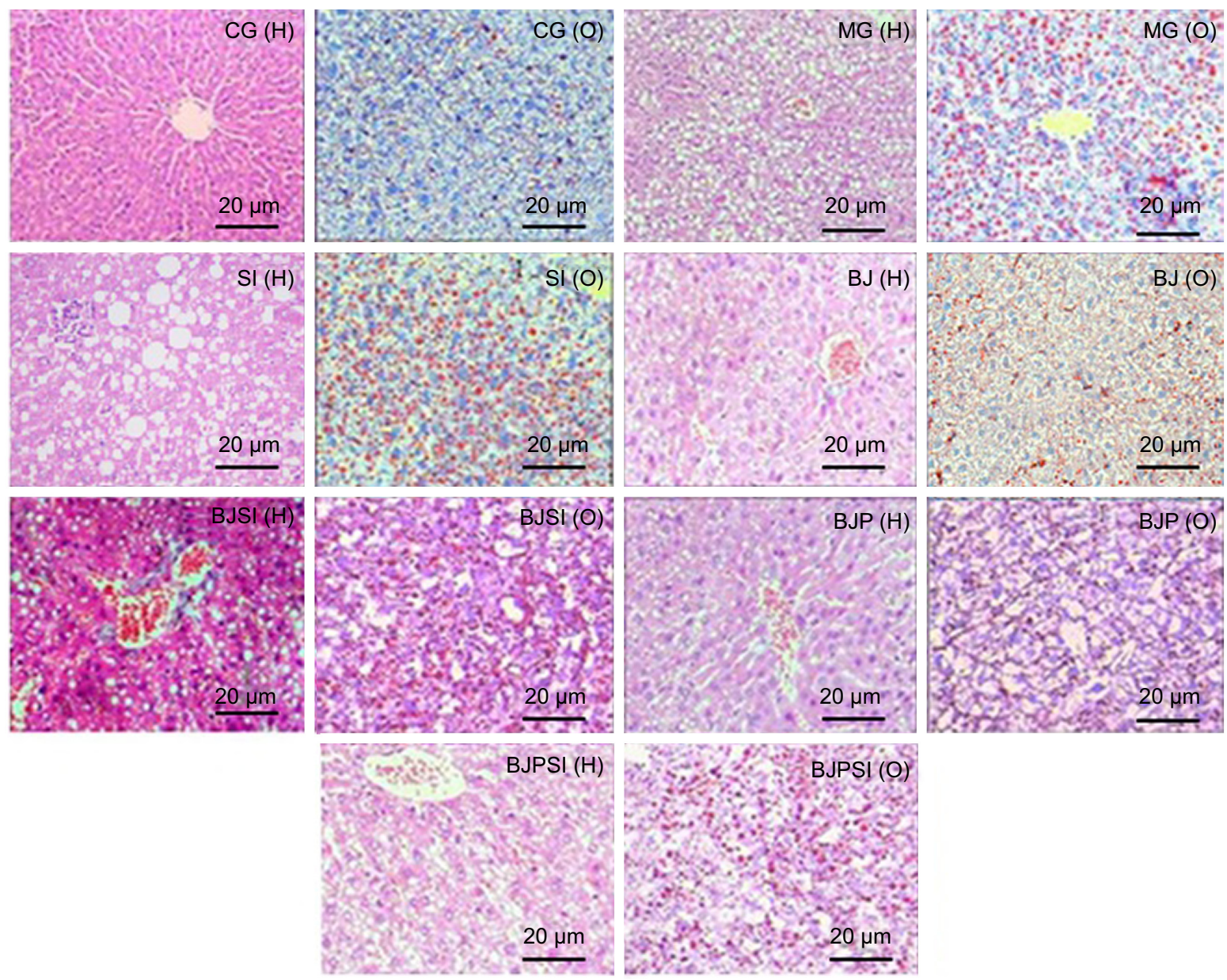

Figure 2 Effect of blueberry juice and probiotics on AFLD and liver damage.

Notes: All hepatic samples were stained with HE (marked as “H”) or Oil Red O (marked as "O") to evaluate the severity of AFLD (original magnification: $\times 200)$. CG (H): in the control group, hepatocytes are arranged radially around a vein in the center and hepatic structure is clearly visible. Few small-sized cavities are found. CG (O): in the control group, small-sized lipid droplets are observed. MG $(\mathrm{H})$ : in the model group, hepatic cords were heavily destroyed and many cavities were observed. MG (O): in the model group, many lipid droplets were visible. SI (H): in the SIRTI-RNAi group, hepatic cords were completely destroyed and many large-sized cavities were produced. SI (O): in the SIRTI-RNAi group, many large-sized lipid droplets were produced. BJ $(\mathrm{H})$ : in the blueberry juice group, the structure of hepatic cords was slightly repaired and arranged around the vein in the center. A few small-sized cavities appeared. BJ $(\mathrm{O})$ : in the blueberry juice group, many small-sized lipid droplets were produced. BJSI $(\mathrm{H})$ : in the blueberry juice and SIRTI-RNAi group, hepatic cords were destroyed and middle-sized cavities were produced. BJSI (O): in the blueberry juice and SIRTI-RNAi group, many middle-sized lipid droplets were found. BJP $(\mathrm{H})$ : in the blueberry juice and probiotics group, the destroyed hepatic cords were repaired well and radially arranged around the vein in the center. Fewer small cavities were produced. BJP (O): in the blueberry juice and probiotics group, fewer small-sized lipid droplets were produced. BJPSI $(\mathrm{H})$ : in the blueberry juice, probiotics, and SIRTI-RNAi group, the hepatic cords were destroyed. Small- and middle-sized cavities were observed. BJPSI (O): in the blueberry juice, probiotics, and SIRTI-RNAi group, many small-sized lipid droplets were produced.

Abbreviations: AFLD, alcoholic fatty liver disease; HE, hematoxylin and eosin; MG, model group; BJ, blueberry juice group; BJSI, blueberry juice and SIRTI siRNA group; $\mathrm{BJP}$, blueberry juice and probiotic bacteria group; BJPSI, blueberry juice, probiotics, and SIRTI siRNA group; RNAi, RNA interference.

\section{Blueberry juice and probiotics improve the biochemical parameters in AFLD mice}

In AFLD model and SI groups, the serum levels of ALT, AST, TGs, TC, and LDL were increased when compared with other groups $(P<0.05)$ (Table 2). Furthermore, the levels of TGs and MDA were also increased in MG and SI groups compared with other groups $(P<0.05)$. In contrast, in AFLD model and SI groups, the serum levels of HDL-C, SOD, and GSH were decreased $(P<0.05)$ (Tables 2 and 3). From these biochemical indexes, the combination therapy was significantly better than the group in which only blueberry juice was used $(P<0.05)$. There was a statistically significant difference between the two treatment groups (BJ and BJP) and the model group $(P<0.05)$.

The results suggested that the combination of blueberry juice and probiotics is better than blueberry intervention alone for AFLD therapy $(P<0.05)$ (Tables 2 and 3$)$. When SIRT1 was silenced, there was no significant statistical difference between the BJSI and MG groups in most cases $(P>0.05)$ (Tables 2 and 3), suggesting that blueberry juice attenuates ALFD by affecting the SIRT1 pathway. There were 


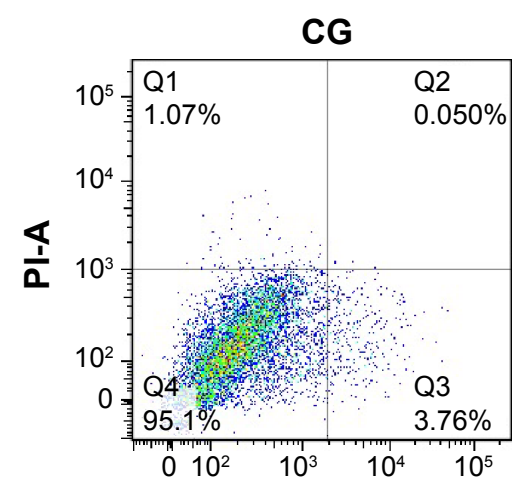

FITC-A:: Annexin V

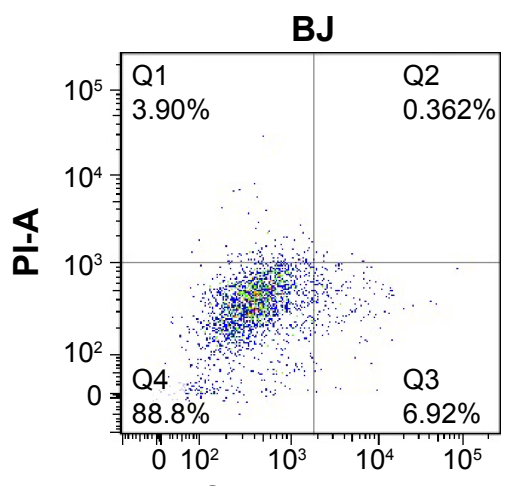

FITC-A:: Annexin V

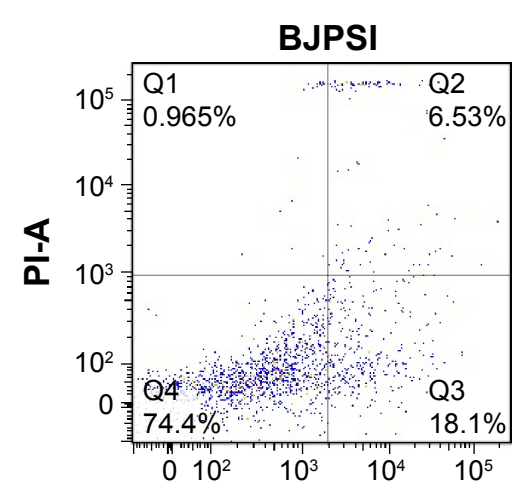

FITC-A:: Annexin V

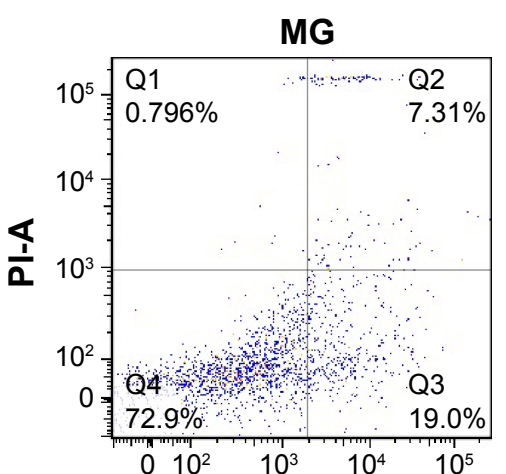

FITC-A:: Annexin V

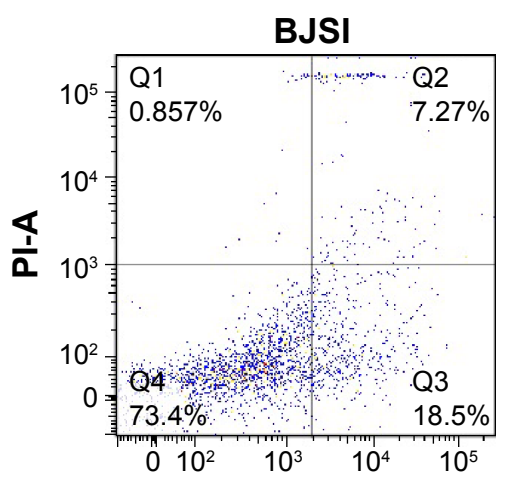

FITC-A:: Annexin V

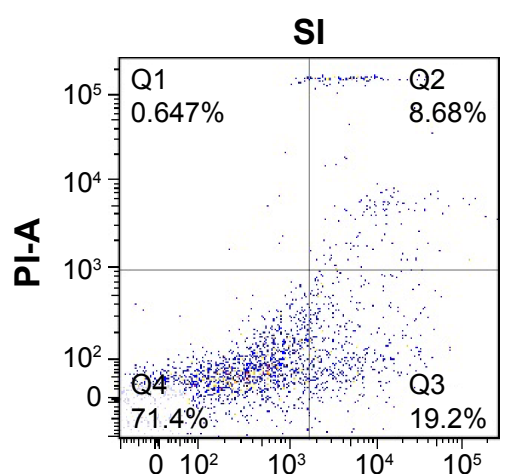

FITC-A:: Annexin V

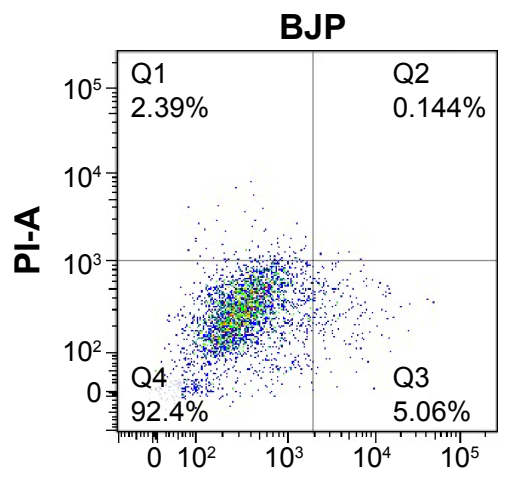

FITC-A:: Annexin V

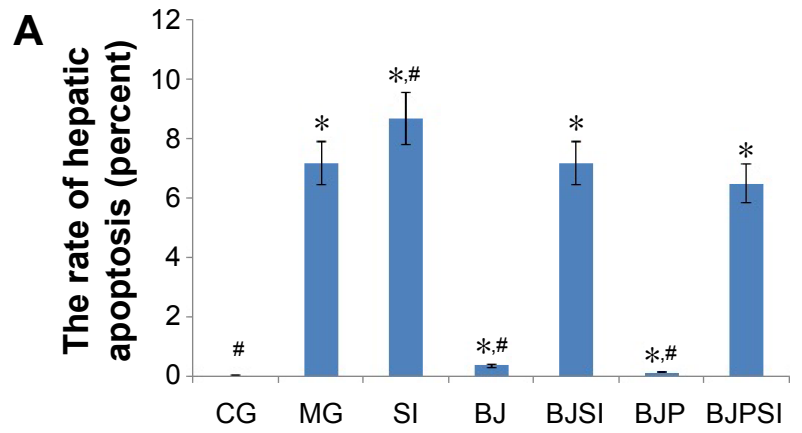

Figure 3 Apoptosis of hepatic tissues.

Notes: CG is the control group, in which the mice were fed with Lieber-DeCarli diet; MG is the model group, in which the mice were fed with Lieber-DeCarli diet and alcohol; SI is a model group, in which SIRTI gene was silenced in the model mice; BJ is a model group, in which the model mice were fed with blueberry juice; BJSI is a model group, in which SIRTI gene was silenced in the model mice and the mice were fed with blueberry juice. BJP is a model group, in which the model mice were fed with blueberry juice and probiotics; BJPSI is a model group, in which SIRTI gene was silenced in model mice and the mice were fed with blueberry juice and probiotics. Panel A shows the apoptotic rates in different groups. All data are presented as mean value \pm SD and $n=8$ in each group. ${ }^{*} P<0.05$ vs control group and ${ }^{\#} P<0.05$ vs model group. ANOVA was performed to compare the mean values from different groups.

Abbreviations: CG, control group; MG, model group; BJ, blueberry juice group; SI, SIRTI siRNA group; SIRTI, sirtuin type I; BJSI, blueberry juice and SIRTI siRNA group; BJP, blueberry juice and probiotic bacteria group; BJPSI, blueberry juice, probiotics, and SIRTI siRNA group; FITC, fluorescein isothiocyanate; SD, standard deviation; PI-A, Propidium lodide-A.

statistically significant differences between BJPSI and MG groups $(P<0.05)$ (Tables 2 and 3$)$, suggesting that blueberry juice and probiotic bacteria still can attenuate ALFD although SIRT1 is silenced. However, the effects were significantly lower than those seen with the SIRT1 gene. Thus, other molecular mechanisms may exist for the synergetic functions of blueberry juice and probiotics.

\section{Relative mRNA levels of SIRTI, FOXOI, FasL, caspase-3, BAX, and Bcl-2}

Real-time qRT-PCR showed that the mRNA levels of SIRT1 were significantly lower in MG, SI, BJSI, and BJPSI groups than in $\mathrm{CG}, \mathrm{BJ}$, and BJP groups $(P<0.05)$ (Figure 4). In contrast, the mRNA levels of FOXO1, FasL, caspase-3, $B A X$, and $B c l-2$ were significantly higher in the MG, SI, 
Table 2 Serum biochemical indexes in all groups

\begin{tabular}{lllllll}
\hline Groups $(\mathbf{n = 8})$ & ALT & AST & TG & TC & HDL & LDL \\
\hline CG & $57.37 \pm I 1.68^{\#}$ & $98.34 \pm 25.47^{\#}$ & $0.73 \pm 0.25^{\#}$ & $1.97 \pm 0.57^{\#}$ & $1.42 \pm 0.22^{\#}$ & $0.89 \pm 0.34^{\#}$ \\
MG & $99.84 \pm 10.48^{*}$ & $326.24 \pm 35.26^{*}$ & $1.78 \pm 0.19^{*}$ & $3.39 \pm 0.68^{*}$ & $0.77 \pm 0.30^{*}$ & $1.45 \pm 0.33^{*}$ \\
SI & $116.21 \pm 5.17^{*, \#}$ & $346.32 \pm 32.79^{*, \#}$ & $1.87 \pm 0.32^{*}$ & $3.25 \pm 0.64^{\#}$ & $0.79 \pm 0.44^{*}$ & $1.57 \pm 0.52^{*, \#}$ \\
BJ & $72.41 \pm 12.58^{*, \#}$ & $126.49 \pm 22.21^{*, \#}$ & $0.86 \pm 0.37^{*, \#}$ & $2.18 \pm 0.3 I^{\#}$ & $1.07 \pm 0.43^{*, \#}$ & $1.03^{*} \pm .18^{*, \#}$ \\
BJSI & $104.25 \pm 14.48^{*, \#}$ & $308.24 \pm 35.26^{*}$ & $1.88 \pm 0.28^{*}$ & $3.56 \pm 0.55^{*}$ & $0.80 \pm 0.28^{*}$ & $1.33^{*} 0.36^{*}$ \\
BJP & $62.37 \pm 8.53^{\#}$ & $107.63 \pm 34.28^{\#}$ & $0.77 \pm 0.23^{\#}$ & $1.88 \pm 0.44^{\#}$ & $1.23 \pm 0.24^{*, \#}$ & $0.86 \pm 0.23^{\#}$ \\
BJPSI & $90.58 \pm 7.48^{*, \#}$ & $288.66 \pm 28.66^{*, \#}$ & $1.73 \pm 0.14^{*}$ & $2.93 \pm 0.68^{*, \#}$ & $0.82 \pm 0.30^{*}$ & $1.26 \pm 0.28^{*, \#}$ \\
\hline
\end{tabular}

Notes: $* P<0.05$ vs CG group; ${ }^{*}<0.05$ vs MG group. All the data are presented as mean $\pm S D(n=8)$. ANOVA was performed to compare the mean values from different groups.

Abbreviations: ALT, alanine aminotransferase; AST, aspartate aminotransferase; TG, total triglyceride; TC, total cholesterol; HDL, high-density lipoprotein; LDL, lowdensity lipoprotein; CG, control group; MG, model group; SI, SIRTI siRNA group; BJ, blueberry juice group; BJSI, blueberry juice and SIRTI siRNA group; BJP, blueberry juice and probiotic bacteria group; BJPSI, blueberry juice, probiotic bacteria, and SIRTI siRNA group.

Table 3 Biochemical indexes of liver tissues

\begin{tabular}{lllll}
\hline Groups $(\mathbf{n}=8)$ & SOD $(\mathbf{U} / \mathbf{m L})$ & MDA $(\mathbf{m m o l} / \mathbf{L})$ & GSH $(\mathbf{n g} / \mathbf{L})$ & TG $(\mathbf{n m o l} / \mathbf{L})$ \\
\hline CG & $27.42 \pm 4.08^{\#}$ & $0.676 \pm 0.332^{\#}$ & $22.415 \pm 2.152^{\#}$ & $1.945 \pm 0.973^{\#}$ \\
MG & $14.26 \pm 5.26^{*}$ & $1.556 \pm 0.276^{*}$ & $14.325 \pm 1.794^{*}$ & $3.586 \pm 0.976^{*}$ \\
SI & $12.67 \pm 4.84^{*}$ & $1.732 \pm 0.394^{*}$ & $12.320 \pm 1.663^{* * \#}$ & $4.843 \pm 0.857^{* \#}$ \\
BJ & $25.67 \pm 4.84^{\#}$ & $0.783 \pm 0.403^{\#}$ & $20.362 \pm 1.773^{\#}$ & $2.145 \pm 0.812^{\#}$ \\
BJSI & $16.31 \pm 4.36^{* * \#}$ & $1.474 \pm 0.472^{*}$ & $13.432 \pm 1.586^{*}$ & $3.472 \pm 1.153^{*}$ \\
BJP & $29.79 \pm 5.79^{\#}$ & $0.525 \pm 0.367^{* * \#}$ & $24.278 \pm 2.667^{\#}$ & $1.813 \pm 0.774^{\#}$ \\
BJPSI & $17.26 \pm 5.26^{* * \#}$ & $1.444 \pm 0.325^{*}$ & $14.356 \pm 1.666^{*}$ & $3.176 \pm 0.852^{* * \#}$ \\
\hline
\end{tabular}

Notes: $* P<0.05$ vs $C G$ group; ${ }^{*}<0.05$ vs $M G$ group. All the data are presented as mean $\pm S D(n=8)$. ANOVA was performed to compare the mean values from different groups.

Abbreviations: SOD, superoxide dismutase; MDA, malondialdehyde; GSH, reduced glutathione; TG, total triglyceride; CG, control group; MG, model group; SD, standard deviation; SI, SIRTI siRNA group; BJ, blueberry juice group; BJSI, blueberry juice and SIRTI siRNA group; BJP, blueberry juice and probiotic bacteria group; BJPSI, blueberry juice, probiotic bacteria, and SIRTI siRNA group.

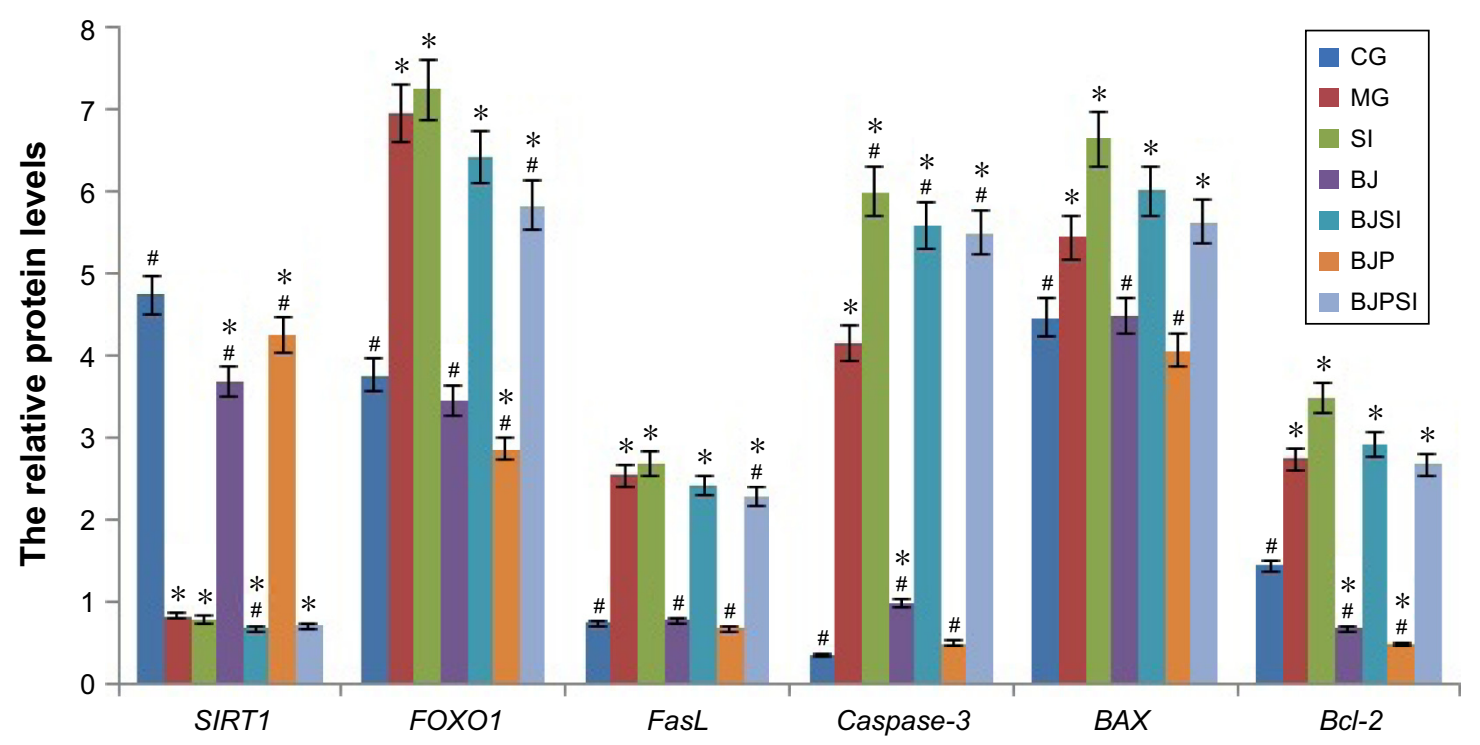

Figure 4 Real-time quantitative RT-PCR analysis of the mRNA levels of SIRTI, FOXOI, FasL, caspase-3, BAX, and BCl-2.

Notes: CG is the control group, in which the mice were fed Lieber-DeCarli diet; MG is the model group, in which the mice were fed Lieber-DeCarli diet and alcohol; SI is a model group, in which SIRTI gene was silenced in the model mice; BJ is a model group, in which the model mice were fed blueberry juice; BJSI is a model group, in which SIRTI gene was silenced in the model mice and the mice were fed blueberry juice. BJP is a model group, in which the model mice were fed blueberry juice and probiotics; BJPSI is a model group, in which SIRTI gene was silenced in model mice and the mice were fed blueberry juice and probiotics. All data are presented as mean value \pm SD and $n=8$ in each group. $* P<0.05$ vs control group and ${ }^{*} P<0.05$ vs $M G$. ANOVA was performed to compare the mean values from different groups.

Abbreviations: BAX, BCL2-associated X protein; Bcl-2, B-cell lymphoma 2; RT-PCR, reverse transcription-polymerase chain reaction; CG, control group; MG, model group; BJ, blueberry juice group; SI, SIRTI siRNA group; SIRTI, sirtuin type I; BJSI, blueberry juice and SIRTI siRNA group; BJP, blueberry juice and probiotic bacteria group; BJPSI, blueberry juice, probiotics, and SIRTI siRNA group; FOXOI, forkhead box protein OI; FasL, tumor necrosis factor ligand superfamily member 6; SD, standard deviation. 
BJSI, and BJPSI groups than in the CG, BJ, and BJP groups $(P<0.05)$ (Figure 4). The results suggest that the combination of blueberry juice and probiotic bacteria increased the mRNA levels of SIRT1 and decreased the levels of FOXO1, FasL, caspase-3, BAX, and Bcl-2. After SIRT1 silencing, the mRNA levels of FOXO1, FasL, caspase-3, BAX, and $B c l-2$ were increased, suggesting that SIRT1 inhibited the mRNA levels of FOXO1, FasL, caspase-3, BAX, and $B c l-2$. Thus, the results confirmed that blueberry juice can inhibit the progression of AFLD by affecting the mRNA level of SIRT1.

\section{Relative protein levels of SIRTI, FOXOI, P-FOXOI,Ac-FOXOI, FasL, caspase-3, $\mathrm{BAX}$, and $\mathrm{Bcl}-2$}

Western blot analysis showed that the protein level of SIRT1 was significantly lower in MG, SI, BJSI, and BJPSI groups than in $\mathrm{CG}, \mathrm{BJ}$, and BJP groups $(P<0.05)$ (Figure 5).
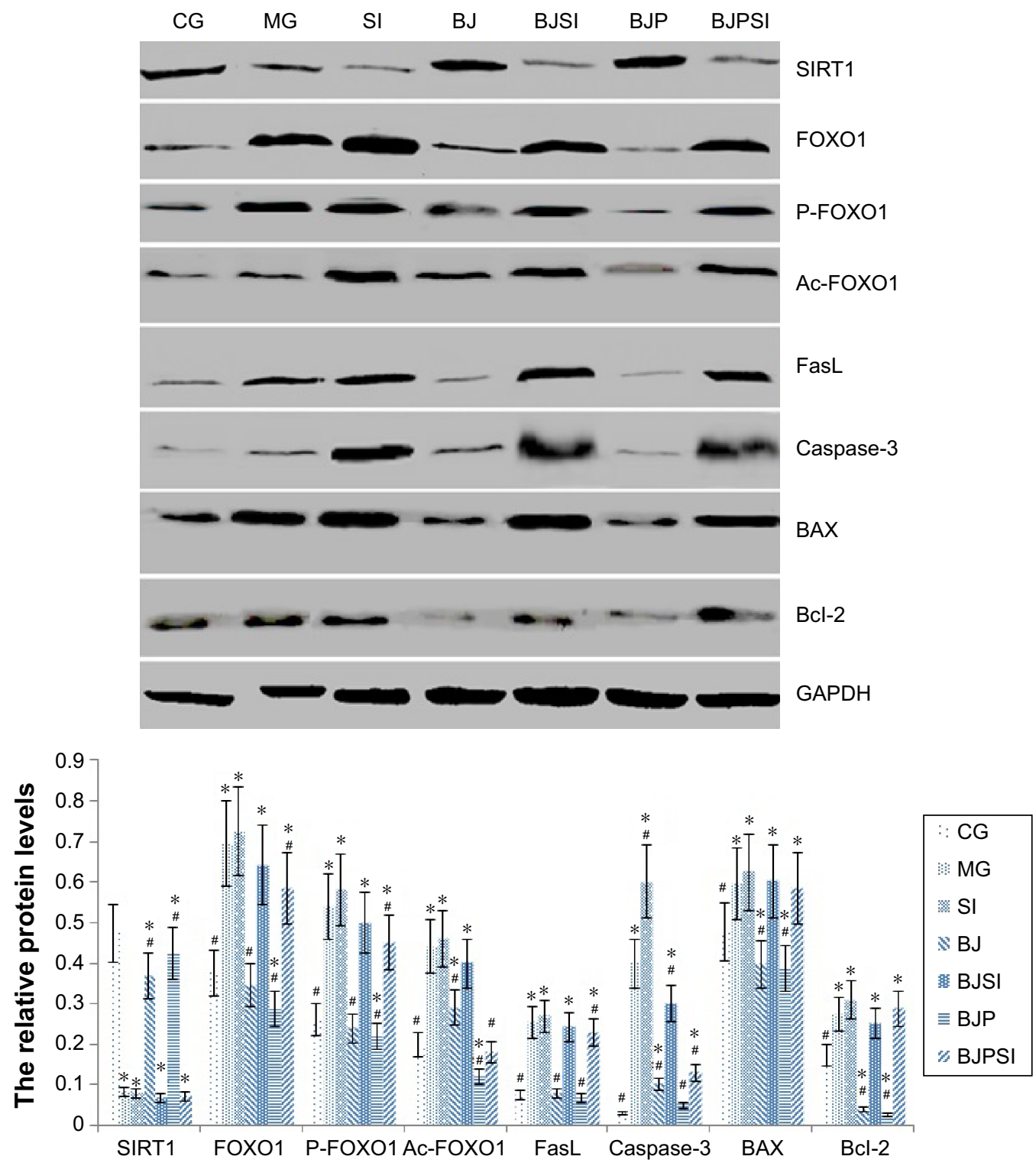

Figure 5 Western blot analysis of the protein levels of SIRTI, FOXOI, phosphorylated FOXOI (P-FOXOI), acetylated-FOXOI (Ac-FOXOI), FasL, and caspase-3. Notes: CG is the control group, in which the mice were fed Lieber-DeCarli diet; MG is the model group, in which the mice were fed Lieber-DeCarli diet and alcohol; SI was the model group, in which SIRTI gene was silenced in the model mice; BJ is a model group, in which the model mice were fed with blueberry juice; BJSI is a model group, in which SIRTI gene was silenced in the model mice and the mice were fed with blueberry juice. BJP is a model group, in which the model mice were fed with blueberry juice and probiotics; BJPSI is a model group, in which SIRTI gene was silenced in model mice and the mice were fed with blueberry juice and probiotics. All data are presented as mean value $\pm S D$ and $n=8$ in each group. $* P<0.05$ vs control group and $\# P<0.05$ vs MG. ANOVA was performed to compare the mean values from different groups.

Abbreviations: CG, control group; MG, model group; BJ, blueberry juice group; SI, SIRTI siRNA group; SIRTI, sirtuin type I; BJSI, blueberry juice and SIRTI siRNA group; $\mathrm{BJP}$, blueberry juice and probiotic bacteria group; BJPSI, blueberry juice, probiotics, and SIRTI siRNA group; FOXOI, forkhead box protein OI; FasL, tumor necrosis factor ligand superfamily member 6; BAX, BCL2-associated X protein; Bcl-2, B-cell lymphoma 2; SD, standard deviation. 
In contrast, the protein levels of FOXO1, P-FOXO1, Ac-FOXO1, FasL, caspase-3, BAX, and Bcl-2 were significantly higher in $\mathrm{MG}, \mathrm{SI}$, BJSI, and BJPSI groups than in $\mathrm{CG}$, BJ, and BJP groups $(P<0.05)$ (Figure 5). The results suggest that the combination of blueberry juice and probiotic bacteria increased the protein levels of SIRT1 and decreased the levels of FOXO1, P-FOXO1, Ac-FOXO1, FasL, caspase-3, BAX, and Bcl-2. After SIRT1 silencing, the protein levels of FOXO1, P-FOXO1, Ac-FOXO1, FasL, caspase-3, BAX, and Bcl-2 were increased, suggesting that SIRT1 inhibited the protein levels of FOXO1, P-FOXO1, Ac-FOXO1, FasL, caspase-3, BAX, and Bcl-2. Thus, the results also confirmed that blueberry juice can inhibit the development of AFLD by affecting the protein level of SIRT1.

\section{Caspase-3 activity}

Lieber-DeCarli diet along with alcohol induced hepatic apoptosis in a mouse model because the activity of caspase- 3 was increased when compared with that in the control group $(P<0.05)$. However, deletion of SIRT1 gene is more likely to result in apoptosis with high activity of caspase- 3 when compared with a model mouse $(P<0.05$, Figure 6$)$, suggesting that caspase- 3 plays an important role in the progression of apoptosis in liver tissues. Comparatively, blueberry juice and/or probiotics decreased the activity of caspase-3 in BJ and BJP groups when compared with the model group, as well as decreasing the activity in BJSI and BJPSI groups

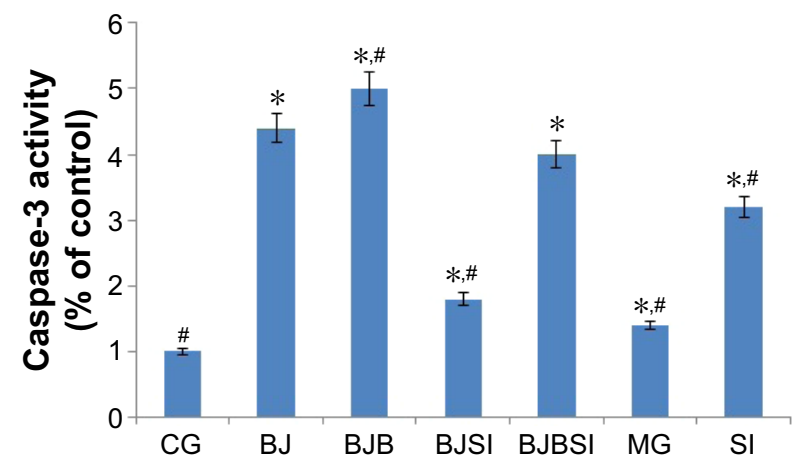

Figure 6 The effects of blueberry juice and probiotics on the activity of caspase- 3 . Notes: CG is the control group, in which the mice were fed Lieber-DeCarli diet; MG is the model group, in which the mice were fed Lieber-DeCarli diet and alcohol; SI was the group, in which SIRTI gene was silenced in the model mice; $\mathrm{BJ}$ is the group, in which the model mice were fed with blueberry juice; BJSI is the group, in which SIRTI gene was silenced in the model mice and the mice were fed with blueberry juice. BJP is the group, in which the model mice were fed with blueberry juice and probiotics; BJPSI is the group, in which SIRTI gene was silenced in model mice and the mice were fed with blueberry juice and probiotics. All data are presented as mean value $\pm S D$ and $n=8$ in each group. $* P<0.05$ vs a control group; ${ }^{*} P<0.05$ vs a model group. ANOVA was performed to compare the mean values from different groups. Abbreviations: CG, control group; MG, model group; BJ, blueberry juice group; SI, SIRTI siRNA group; SIRTI, sirtuin type I; BJSI, blueberry juice and SIRTI siRNA group; BJP, blueberry juice and probiotic bacteria group; BJPSI, blueberry juice, probiotics, and SIRTI siRNA group; SD, standard deviation. when compared with the SI group ( $P<0.05$, Figure 6), confirming that blueberry juice and/or probiotics prevent apoptosis by inhibiting the activity of caspase-3.

\section{Discussion}

Oxidative stress is the main reason for the pathogenesis of AFLD. Alcohol metabolism often produces more oxygen free radicals (reactive oxygen species [ROS]), which lead to hepatic apoptosis. ${ }^{8}$ Thus, it is important to prevent ROS production in hepatocytes at an early stage and maintain the balance between proliferation and apoptosis of hepatocytes. This study reports the pathogenesis of AFLD and shows the destruction of hepatic tissues because of the changes in antioxidant enzymes. The combination of blueberry juice and probiotic bacteria significantly reduced lipid deposition, inflammation, necrosis, and fibrosis in hepatic tissues by enhancing the activity of the enzyme SOD and decreasing the levels of ALT and AST.

Flow cytometry analysis showed that the combination of blueberry juice and probiotic bacteria had a significant inhibitory effect on liver cell apoptosis. The apoptotic rate in the BJP group was lower than that in the BJ group $(P<0.05)$ (Figure 3A), suggesting that there is a synergistic effect of the combination of blueberry juice and probiotic bacteria during AFLD therapy. However, the detailed molecular mechanisms for the collaborative functions still need to be further confirmed in the future.

To elucidate the molecular mechanism of our results, some important molecules were explored. SIRT1, a powerful nicotinamide adenine dinucleotide (NAD+)-dependent histone deacetylase, ${ }^{55}$ plays an important role in deacetylation, is involved in cell apoptosis, cell cycle regulation, gene transcription, metabolism, and many other cellular processes, and is associated with the incidence and progression of AFLD. SIRT1 regulates the ratio of NAD/NADH. ${ }^{56}$ Blueberry juice shows antioxidant activity and inhibits oxidative damage by reducing the ratio of NAD/NADH. ${ }^{57}$ In addition, blueberry juice contains the polyphenol resveratrol, which is currently found to increase the SIRT1 levels up to eight fold. ${ }^{58}$ This study demonstrated that, compared with the model group, blueberry juice and probiotic bacteria enhance SIRT1 expression and then affect the downstream effects of the SIRT1 pathway.

The balance between oxidative and antioxidant pathways plays a vital role in protecting the liver cells from damage. SOD is regulated by SIRT $1 .{ }^{59}$ Therefore, blueberry improves SIRT1 activity, which increases SOD level, resulting in a decrease in free radical levels. On the other hand, as a 
downstream factor in SIRT1 regulation, FOXO1 plays an important role in regulating mammalian apoptosis, oxidative stress, and cell cycle. FOXO1 is a transcription factor, and its downregulation can inhibit the expression of FasL. FasL induces the damage and the apoptosis of liver. ${ }^{58}$ FasL can activate caspase, which increases the activity of DNAdegrading enzymes and leads to the degradation of DNA, resulting in liver apoptosis. ${ }^{60}$ In this study, the levels of FOXO1, P-FOXO1, Ac-FOXO1, FasL, caspase-3, BAX, and $\mathrm{Bcl}-2$ were significantly lower in the BJ and BJP groups than in the model group, suggesting that the intervention of blueberry juice and probiotics activates the SIRT1 gene, which downregulates the apoptotic factors FOXO1, P-FOXO1, and Ac-FOXO1, resulting in the decrease of the levels of FasL, caspase-3, BAX, and Bcl-2. All these result in the inhibition of the progression of hepatocyte apoptosis and prevent excessive necrosis of hepatocytes.

To prove the earlier theory, lentivirus-packaged SIRT1 siRNA was used to infect C57/6J mouse livers. The levels of FOXO1, P-FOXO1, Ac-FOXO1, FasL, caspase-3, BAX, and Bcl-2 were changed significantly and there were significant differences between the BJ and the BJSI groups after SIRT1 silencing. On the other hand, there were significant differences in the apoptotic rates between the SI and the BJSI/BJPSI groups (Figure 3). Furthermore, the combination of blueberry juice and probiotic bacteria could still ameliorate the severity of AFLD even after SIRT1 was silenced. Therefore, these findings confirm that there may be other molecular mechanisms for the inhibition of the severity of fatty liver by blueberry juice and probiotics. The results suggest that probiotic bacteria also play an important regulatory role in the protection of the liver from damage caused by alcohol. Probiotic bacteria and blueberry juice can synergistically enhance the protective effects However, the exact mechanism remains to be confirmed by further studies.

Certainly, there are some limitations in this study. First, SIRT1 RNA interference was not performed in healthy mice. There are two reasons: some conclusions can be arrived at only using model mice without SIRT1 gene silencing as controls; to reduce injury to animals, only limited mice were used for this study according to the instructions of our animal research and ethical committee of the Suzhou University. Second, there are complex components in the combination of blueberry juice and probiotics, which leads to difficulty in clearly explaining the molecular mechanism of dual therapy. According to our previous experiments, it is hard to obtain good therapeutic results if only one main component of blueberry juice or one type of probiotic was used (Zhu et al, unpublished data, 2015). Thus, there is still a conflict regarding how this study should have been designed. Finally, clinical trials were not performed, but we are sure that there are no harmful effects on humans. Much evidence needs to be accumulated before conducting such an experiment in patients with AFLD in the future.

\section{Conclusion}

This study clearly showed that the combination of blueberry juice and probiotic bacteria has a synergistic effect against the progression of AFLD. Blueberry juice can reduce the damage and apoptosis of AFLD by improving the activity of SIRT1 and promote activity of liver cells against oxidative damage by increasing the activities of related enzymes to remove oxygen free radicals, protect liver cells, effectively prevent lipid peroxidation, and regulate lipid metabolism. On the other hand, increase in SIRT1 downregulates the levels of FOXO1, P-FOXO1, and Ac-FOXO1, resulting in the inhibition of FasL, caspase-3, BAX, and Bcl-2. In addition, SIRT1 siRNA proves that blueberry juice reduces the apoptosis of hepatocytes in AFLD by affecting the SIRT1 pathway, which plays an important role in the incidence and progression of AFLD.

\section{Disclosure}

The authors report no conflicts of interest in this work.

\section{References}

1. Lee WS, Kim TY. Alcoholic fatty liver disease and alcoholic liver cirrhosis may be differentiated with mean platelet volume and platelet distribution width. Platelets. 2010;21(7):584-585.

2. Ishikawa $T$, Tachibana $T$, Ishikawa $H$, Miyaishi S, Ishizu H. Increase of S-100 protein-positive stellate cells in the anterior pituitary of chronic alcoholic patients with fatty liver or fatty cirrhosis. Acta Med Okayama. 2003;57(2):53-58.

3. Lieber CS. Alcoholic fatty liver: its pathogenesis and mechanism of progression to inflammation and fibrosis. Alcohol. 2004;34(1): 9-19.

4. Rehm J, Samokhvalov AV, Shield KD. Global burden of alcoholic liver diseases. J Hepatol. 2013;59(1):160-168.

5. Hmoud BS, Patel K, Bataller R, Singal AK. Corticosteroids and occurrence of and mortality from infections in severe alcoholic hepatitis: a meta-analysis of randomized trials. Liver Int. Epub 2015 Aug 17.

6. Poynard T, Thabut D, Chryssostalis A, Taieb J, Ratziu V. Anti-tumor necrosis factor-alpha therapy in severe alcoholic hepatitis: are large randomized trials still possible? J Hepatol. 2003;38(4):518-520.

7. Gao B, Bataller R. Alcoholic liver disease: pathogenesis and new therapeutic targets. Gastroenterology. 2011;141(5):1572-1585.

8. Feldstein AE, Bailey SM. Emerging role of redox dysregulation in alcoholic and nonalcoholic fatty liver disease. Antioxid Redox Signal. 2011;15(2):421-424.

9. Farinati F, Cardin R, Fiorentino M. Apoptosis in alcoholic hepatitis. J Hepatol. 2002;36(4):570-571.

10. Lin FS, Shen SQ, Chen ZB, Yan RC. 17beta-estradiol attenuates reduced-size hepatic ischemia/reperfusion injury by inhibition apoptosis via mitochondrial pathway in rats. Shock. 2012;37(2):183-190. 
11. Liu J, Zhou J, Wu Z, Wang X, Liu L, Yao C. Cyanidin 3-O-beta-Glucoside Ameliorates Ethanol-Induced Acute Liver Injury by Attenuating Oxidative Stress and Apoptosis: The Role of SIRT1/FOXO1 Signaling. Alcohol Clin Exp Res. 2016;40(3):457-466.

12. Luo M, Zhao A, Li J, et al. Acute liver injury attenuation of a novel recombinant sTNFR through blocking hepatic apoptosis. Immunopharmacol Immunotoxicol. 2015;37(3):295-300.

13. Li G, Ye Y, Kang J, et al. 1-Theanine prevents alcoholic liver injury through enhancing the antioxidant capability of hepatocytes. Food Chem Toxicol. 2012;50(2):363-372.

14. Barberis A, Spissu Y, Fadda A, et al. Simultaneous amperometric detection of ascorbic acid and antioxidant capacity in orange, blueberry and kiwi juice, by a telemetric system coupled with a fullerene- or nanotubes-modified ascorbate subtractive biosensor. Biosens Bioelectron. 2015;67:214-223.

15. Kraujalyte V, Venskutonis PR, Pukalskas A, Cesoniene L, Daubaras R. Antioxidant properties, phenolic composition and potentiometric sensor array evaluation of commercial and new blueberry (Vaccinium corymbosum) and bog blueberry (Vaccinium uliginosum) genotypes. Food Chem. 2015;188:583-590.

16. Nayak BN, Buttar HS. Evaluation of the antioxidant properties of tryptophan and its metabolites in in vitro assay. J Complement Integr Med. Epub 2015 Dec 8.

17. Kamat JP, Devasagayam TP. Nicotinamide (vitamin B3) as an effective antioxidant against oxidative damage in rat brain mitochondria. Redox Rep. 1999;4(4):179-184.

18. Pero RW, Lund H, Leanderson T. Antioxidant metabolism induced by quinic acid. Increased urinary excretion of tryptophan and nicotinamide. Phytother Res. 2009;23(3):335-346.

19. Zhou Y, Zhou L, Ruan Z, et al. Chlorogenic acid ameliorates intestinal mitochondrial injury by increasing antioxidant effects and activity of respiratory complexes. Biosci Biotechnol Biochem. Epub 2016 Jan29:1-10.

20. Samsonowicz M, Kaminska I, Kalinowska M, Lewandowski W. Alkali metal salts of rutin - synthesis, spectroscopic (FT-IR, FT-Raman, UV-VIS), antioxidant and antimicrobial studies. Spectrochim Acta A Mol Biomol Spectrosc. 2015;151:926-938.

21. Gioacchini G, Giorgini E, Olivotto I, Maradonna F, Merrifield DL, Carnevali $O$. The influence of probiotics on zebrafish Danio rerio innate immunity and hepatic stress. Zebrafish. 2014;11(2):98-106.

22. Li F, Duan K, Wang C, McClain C, Feng W. Probiotics and alcoholic liver disease: treatment and potential mechanisms. Gastroenterol Res Pract. 2016;2016:5491465.

23. Liu GZ, Hou TT, Yuan Y, et al. Fenofibrate inhibits atrial metabolic remodeling in atrial fibrillation through PPAR-alpha/Sirt1/PGC-1alpha pathway. Br J Pharmacol. Epub 2016 Jan 20.

24. Zhao S, Li T, Li J, et al. miR-23b-3p induces the cellular metabolic memory of high glucose in diabetic retinopathy through a SIRT1dependent signalling pathway. Diabetologia. 2016;59(3):644-654.

25. Li X, Lian F, Liu C, Hu KQ, Wang XD. Isocaloric pair-fed highcarbohydrate diet induced more hepatic steatosis and inflammation than high-fat diet mediated by miR-34a/SIRT1 axis in mice. Sci Rep. 2015;5:16774.

26. Choi YH, Bae JK, Chae HS, et al. $\alpha$-Mangostin regulates hepatic steatosis and obesity through SirT1-AMPK and PPARgamma pathways in high-fat diet-induced obese mice. J Agric Food Chem. 2015; 63(38):8399-8406.

27. Mariani S, Fiore D, Basciani S, et al. Plasma levels of SIRT1 associate with non-alcoholic fatty liver disease in obese patients. Endocrine. 2015;49(3):711-716.

28. Li X. SIRT1 and energy metabolism. Acta Biochim Biophys Sin (Shanghai). 2013;45(1):51-60.

29. Quinn LS, Anderson BG, Conner JD, Wolden-Hanson T. IL-15 overexpression promotes endurance, oxidative energy metabolism, and muscle PPARdelta, SIRT1, PGC-1alpha, and PGC-1beta expression in male mice. Endocrinology. 2013;154(1):232-245.

30. Wei W, Li L, Zhang Y, et al. Vitamin C protected human retinal pigmented epithelium from oxidant injury depending on regulating SIRT1. ScientificWorldJournal. 2014;2014:750634
31. Zheng T, Lu Y. SIRT1 protects human lens epithelial cells against oxidative stress by inhibiting p53-dependent apoptosis. Curr Eye Res. Epub 2015 Dec 30:1-8.

32. Zhou S, Chen HZ, Wan YZ, et al. Repression of P66Shc expression by SIRT1 contributes to the prevention of hyperglycemia-induced endothelial dysfunction. Circ Res. 2011;109(6):639-648.

33. Xu X, Hu Y, Zhai X, et al. Salvianolic acid A preconditioning confers protection against concanavalin A-induced liver injury through SIRT1mediated repression of p66shc in mice. Toxicol Appl Pharmacol. 2013; 273(1):68-76.

34. Miyazaki S, Kakutani K, Yurube T, et al. Recombinant human SIRT1 protects against nutrient deprivation-induced mitochondrial apoptosis through autophagy induction in human intervertebral disc nucleus pulposus cells. Arthritis Res Ther. 2015;17:253.

35. Wu H, Wang $\mathrm{H}$, Zhang $\mathrm{W}$, et al. rhEPO affects apoptosis in hippocampus of aging rats by upregulating SIRT1. Int J Clin Exp Pathol. 2015;8(6):6870-6880.

36. Liu Z, Chen B, He M, Zhang X, Wang H, Hu B. Application of inductively coupled plasma mass spectrometry in the study of apoptosis: determination of caspase-3 using a gold nanoparticle tag. Analyst. 2016;141(3):926-933.

37. Cohen HY, Miller C, Bitterman KJ, et al. Calorie restriction promotes mammalian cell survival by inducing the SIRT1 deacetylase. Science. 2004;305(5682):390-392.

38. Kuo SJ, Lin HY, Chien SY, Chen DR. SIRT1 suppresses breast cancer growth through downregulation of the Bcl-2 protein. Oncol Rep. 2013;30(1):125-130.

39. Ning Y, Li Z, Qiu Z. FOXO1 silence aggravates oxidative stresspromoted apoptosis in cardiomyocytes by reducing autophagy. J Toxicol Sci. 2015;40(5):637-645.

40. Punsawad C, Viriyavejakul P, Setthapramote C, Palipoch S. Enhanced expression of Fas and FasL modulates apoptosis in the lungs of severe $P$. falciparum malaria patients with pulmonary edema. Int J Clin Exp Pathol. 2015;8(9):10002-10013.

41. Law NC, Weck J, Kyriss B, Nilson JH, Hunzicker-Dunn M. Lhcgr expression in granulosa cells: roles for PKA-phosphorylated beta-catenin, TCF3, and FOXO1. Mol Endocrinol. 2013;27(8):1295-1310.

42. Wang WR, Liu EQ, Zhang JY, et al. Activation of PPAR alpha by fenofibrate inhibits apoptosis in vascular adventitial fibroblasts partly through SIRT1-mediated deacetylation of FoxO1. Exp Cell Res. 2015;338(1):54-63.

43. GuangQin W, Fei N, YouJiang L. Comparative analysis of physicochemical component content in blueberry fruit and their function evaluation. Acta Agriculturae Jiangxi. 2012;24(1):117-119.

44. Tan M, Zhu JC, Du J, Zhang LM, Yin HH. Effects of probiotics on serum levels of Th1/Th2 cytokine and clinical outcomes in severe traumatic brain-injured patients: a prospective randomized pilot study. Crit Care. 2011;15(6):R290.

45. Wang Y, Cheng M, Zhang B, Wu J. [Effects of blueberry on the expression of PPAR $\gamma$ and PDGF-B in rat hepatic fibrosis]. Zhonghua Yi Xue Za Zhi. 2013;93(3):218-221.

46. Chi X, Zhang R, Shen N, et al. Sulforaphane reduces apoptosis and oncosis along with protecting liver injury-induced ischemic reperfusion by activating the Nrf2/ARE pathway. Hepatol Int. 2015;9(2): 321-329.

47. Łuszczak J, Ziaja-Sołtys M, Rzymowska J. Anti-oxidant activity of superoxide dismutase and glutathione peroxidase enzymes in skeletal muscles from slaughter cattle infected with Taenia saginata. Exp Parasitol. 2011;128(2):163-165.

48. Everitt H, Hu M, Ajmo JM, et al. Ethanol administration exacerbates the abnormalities in hepatic lipid oxidation in genetically obese mice. Am J Physiol Gastrointest Liver Physiol. 2013;304(1):G38-G47.

49. Kumar P, Maurya PK. L-cysteine efflux in erythrocytes as a function of human age: correlation with reduced glutathione and total anti-oxidant potential. Rejuvenation Res. 2013;16(3):179-184.

50. Kumawat M, Kharb S, Singh V, Singh N, Singh SK, Nada M. Plasma malondialdehyde (MDA) and anti-oxidant status in diabetic retinopathy. J Indian Med Assoc. 2014;112(1):29-32. 
51. Paoletti F, Mocali A. Determination of superoxide dismutase activity by purely chemical system based on NAD(P)H oxidation. Methods Enzymol. 1990;186:209-220.

52. Hissin PJ, Hilf R. A fluorometric method for determination of oxidized and reduced glutathione in tissues. Anal Biochem. 1976;74(1): 214-226.

53. Gastaldelli A, Gaggini M, Mezzabotta L, et al. Alteration in lipid metabolism after an oral fat load in subjects with NAFLD. Diabetologia. 2013;56:S323-S323.

54. Musso G, Gambino R, Cassader M. Recent insights into hepatic lipid metabolism in non-alcoholic fatty liver disease (NAFLD). Prog Lipid Res. 2009;48(1):1-26.

55. Lin CS, Chang CJ, Lu CC, et al. Impact of the gut microbiota, prebiotics, and probiotics on human health and disease. Biomed $J$. 2014;37(5):259-268.

56. Huang J, Yue S, Ke B, et al. Nuclear factor erythroid 2-related factor 2 regulates toll-like receptor 4 innate responses in mouse liver ischemiareperfusion injury through Akt-forkhead box protein O1 signaling network. Transplantation. 2014;98(7):721-728.
57. Suhara T, Kim HS, Kirshenbaum LA, Walsh K. Suppression of Akt signaling induces Fas ligand expression: involvement of caspase and Jun kinase activation in Akt-mediated Fas ligand regulation. Mol Cell Biol. 2002;22(2):680-691.

58. Tsalkidou EG, Tsaroucha AK, Chatzaki E, et al. The effects of apigenin on the expression of Fas/FasL apoptotic pathway in warm liver ischemiareperfusion injury in rats. Biomed Res Int. 2014;2014:157216.

59. Su S, Li Q, Liu Y, et al. Sesamin ameliorates doxorubicin-induced cardiotoxicity: involvement of Sirt1 and Mn-SOD pathway. Toxicol Lett. 2014;224(2):257-263.

60. Wall DM, McCormick BA. Bacterial secreted effectors and caspase-3 interactions. Cell Microbiol. 2014;16(12):1746-1756.

\section{Publish your work in this journal}

Drug Design, Development and Therapy is an international, peerreviewed open-access journal that spans the spectrum of drug design and development through to clinical applications. Clinical outcomes, patient safety, and programs for the development and effective, safe, and sustained use of medicines are a feature of the journal, which has also been accepted for indexing on PubMed Central. The manuscript management system is completely online and includes a very quick and fair peer-review system, which is all easy to use. Visit http://www.dovepress.com/testimonials.php to read real quotes from published authors.

Submit your manuscript here: http://www.dovepress.com/drug-design-development-and-therapy-journal 